\title{
Henry-Gronwall Integral Inequalities with "Maxima" and Their Applications to Fractional Differential Equations
}

\author{
Phollakrit Thiramanus, ${ }^{1,2}$ Jessada Tariboon, ${ }^{1,2}$ and Sotiris K. Ntouyas ${ }^{3}$ \\ ${ }^{1}$ Nonlinear Dynamic Analysis Research Center, Department of Mathematics, Faculty of Applied Science, King Mongkut's University of \\ Technology North Bangkok, Bangkok 10800, Thailand \\ ${ }^{2}$ Centre of Excellence in Mathematics, CHE, Si Ayutthaya Road, Bangkok 10400, Thailand \\ ${ }^{3}$ Department of Mathematics, University of Ioannina, 45110 Ioannina, Greece
}

Correspondence should be addressed to Jessada Tariboon; jessadat@kmutnb.ac.th

Received 25 April 2014; Revised 9 June 2014; Accepted 9 June 2014; Published 25 June 2014

Academic Editor: Dumitru Baleanu

Copyright (C) 2014 Phollakrit Thiramanus et al. This is an open access article distributed under the Creative Commons Attribution License, which permits unrestricted use, distribution, and reproduction in any medium, provided the original work is properly cited.

Some new weakly singular Henry-Gronwall type integral inequalities with "maxima" are established in this paper. Applications to Caputo fractional differential equations with "maxima" are also presented.

\section{Introduction}

It is well known that Gronwall-Bellman type integral inequalities play a dominant role in the study of quantitative properties of solutions of differential and integral equations [1-5]. Usually, the integrals concerning these type inequalities have regular or continuous kernels, but some problems of theory and practicality require us to solve integral inequalities with singular kernels. For example, Henry [6] proposed a method to find solutions and proved some results concerning linear integral inequalities with weakly singular kernel. Moreover, Medved' [7, 8] presented a new approach to solve integral inequalities of Henry-Gronwall type and their Bihari version and obtained global solutions of semilinear evolution equations. Ye and Gao [9] considered the integral inequalities of Henry-Gronwall type and their applications to fractional differential equations with delay. Ma and Pečarić [10] established some weakly singular integral inequalities of Gronwall-Bellman type and used them in the analysis of various problems in the theory of certain classes of differential equations, integral equations, and evolution equations. Shao and Meng [11] studied a certain class of nonlinear inequalities of Gronwall-Bellman type, which is used to a qualitative analysis to certain fractional differential equations. For other results on the subject we refer to [12-18] and references cited therein.
Differential equations with "maxima" are a special type of differential equations that contain the maximum of the unknown function over a previous interval. Several integral inequalities have been established in the case when maxima of the unknown scalar function are involved in the integral; see $[19,20]$ and references cited therein.

Recently in [21] some new types of integral inequalities on time scales with "maxima" are established, which can be used as a handy tool in the investigation of making estimates for bounds of solutions of dynamic equations on time scales with "maxima." In this paper we establish some Henry-Gronwall type integral inequalities with "maxima." The significance of our work lies in the fact that "maxima" are taken on intervals $[\beta t, t]$ which have nonconstant length, where $0<\beta<1$. Most of the papers take the "maxima" on $[t-h, t]$, where $h>0$ is a given constant. We apply our results to demonstrate the bound of solutions and the dependence of solutions on the orders with initial conditions for Caputo fractional differential equations with "maxima"

$$
\begin{gathered}
D^{\alpha} x(t)=f\left(t, x(t), \max _{s \in[\beta t, t]} x(s)\right), \quad t \in I=\left[t_{0}, T\right), \\
x(t)=\phi(t), \quad t \in\left[\beta t_{0}, t_{0}\right] .
\end{gathered}
$$

The paper is organized as follows. In Section 2 we recall some results from [21] in the special case $\mathbb{T}=\mathbb{R}$ which 
are used to prove our main results which are presented in Section 3. In the last Section 4 we give applications of our results for an initial value problem for a Caputo fractional differential equation with "maxima."

\section{Preliminaries}

For convenience we let throughout $t_{0}>0$. The following results in Lemmas 1 and 3 are obtained by reducing the time scales $\mathbb{T}=\mathbb{R}, f(t)=g(t) \equiv 1$, and $a(t)=b(t) \equiv 0$ for all $t \in\left[t_{0}, T\right)$ in Theorems 3.3 and 3.2 ([21], page 8 and page 6$)$, respectively.

Lemma 1 (see [21]). Let the following conditions be satisfied:

$\left(H_{1}\right)$ the functions $p$ and $q \in C\left(\left[t_{0}, T\right), \mathbb{R}_{+}\right)$;

$\left(H_{2}\right)$ the function $\phi \in C\left(\left[\beta t_{0}, T\right), \mathbb{R}_{+}\right)$with $\max _{s \in\left[\beta t_{0}, t_{0}\right]} \phi(s)>0$, where $0<\beta<1$;

$\left(H_{3}\right)$ the function $u \in C\left(\left[\beta t_{0}, T\right), \mathbb{R}_{+}\right)$and satisfies the inequalities

$$
\begin{gathered}
u(t) \leq \phi(t)+\int_{t_{0}}^{t}\left[p(s) u(s)+q(s) \max _{\xi \in[\beta s, s]} u(\xi)\right] d s, \\
t \in\left[t_{0}, T\right], \\
u(t) \leq \phi(t), \quad t \in\left[\beta t_{0}, t_{0}\right] .
\end{gathered}
$$

Then

$$
u(t) \leq \phi(t)+h(t) \exp \left(\int_{t_{0}}^{t}[p(s)+q(s)] d s\right), \quad t \in\left[t_{0}, T\right)
$$

holds, where

$$
\begin{array}{r}
h(t)=\max _{s \in\left[\beta t_{0}, t_{0}\right]} \phi(s)+\int_{t_{0}}^{t}\left[p(s) \phi(s)+q(s) \max _{\xi \in[\beta s, s]} \phi(\xi)\right] d s, \\
t \in\left[t_{0}, T\right) .
\end{array}
$$

By splitting the initial function $\phi$ to be two functions, we deduce the following corollary.

Corollary 2. Let the following conditions be satisfied:

$\left(H_{4}\right)$ the functions $p, q$, and $v \in C\left(\left[t_{0}, T\right), \mathbb{R}_{+}\right)$;

$\left(H_{5}\right)$ the function $w \in C\left(\left[\beta t_{0}, t_{0}\right], \mathbb{R}_{+}\right)$with $\max _{s \in\left[\beta t_{0}, t_{0}\right]} w(s)>0$ and $w\left(t_{0}\right)=v\left(t_{0}\right)$, where $0<\beta<1$

$\left(H_{6}\right)$ the function $u \in C\left(\left[\beta t_{0}, T\right), \mathbb{R}_{+}\right)$and satisfies the inequalities

$$
\begin{array}{r}
u(t) \leq v(t)+\int_{t_{0}}^{t}\left[p(s) u(s)+q(s) \max _{\xi \in[\beta s, s]} u(\xi)\right] d s, \\
t \in\left[t_{0}, T\right),
\end{array}
$$

Then

$$
\begin{array}{r}
u(t) \leq v(t)+h(t) \exp \left(\int_{t_{0}}^{t}[p(s)+q(s)] d s\right), \\
t \in\left[t_{0}, T\right)
\end{array}
$$

holds, where

$$
\begin{array}{r}
h(t)=\max _{s \in\left[\beta t_{0}, t_{0}\right]} w(s)+\int_{t_{0}}^{t}\left[p(s) v(s)+q(s) \max _{\xi \in[\beta s, s]} m(\xi)\right] d s, \\
t \in\left[t_{0}, T\right),
\end{array}
$$

with

$$
m(t)= \begin{cases}v(t), & t \in\left[t_{0}, T\right) \\ w(t), & t \in\left[\beta t_{0}, t_{0}\right]\end{cases}
$$

Lemma 3 (see [21]). Let the condition $\left(H_{1}\right)$ of Lemma 1 be satisfied. In addition, assume that

$\left(H_{7}\right)$ the function $k \in C\left(\left[t_{0}, T\right),(0, \infty)\right)$ is nondecreasing;

$\left(H_{8}\right)$ the function $\phi \in C\left(\left[\beta t_{0}, t_{0}\right), \mathbb{R}_{+}\right)$, where $0<\beta<1$;

$\left(H_{9}\right)$ the function $u \in C\left(\left[\beta t_{0}, T\right), \mathbb{R}_{+}\right)$and satisfies the inequalities

$$
\begin{gathered}
u(t) \leq k(t)+\int_{t_{0}}^{t}\left[p(s) u(s)+q(s) \max _{\xi \in[\beta s, s]} u(\xi)\right] d s, \\
t \in\left[t_{0}, T\right), \\
u(t) \leq \phi(t), \quad t \in\left[\beta t_{0}, t_{0}\right] .
\end{gathered}
$$

Then

$$
u(t) \leq N k(t) \exp \left(\int_{t_{0}}^{t}[p(s)+q(s)] d s\right), \quad t \in\left[t_{0}, T\right)
$$

holds, where

$$
N=\max \left\{1, \frac{\max _{s \in\left[\beta t_{0}, t_{0}\right]} \phi(s)}{k\left(t_{0}\right)}\right\} .
$$

The following lemma is a consequence of Jensen's inequality which can be found in [22].

Lemma 4 (see [22]). Let $n \in N$, and let $x_{1}, \ldots, x_{n}$ be nonnegative real numbers. Then, for $\sigma>1$,

$$
\left(\sum_{i=1}^{n} x_{i}\right)^{\sigma} \leq n^{\sigma-1} \sum_{i=1}^{n} x_{i}^{\sigma}
$$

\section{Main Results}

Theorem 5. Suppose that the following conditions are satisfied:

$\left(H_{10}\right)$ the functions $p$ and $r \in C\left(\left[t_{0}, T\right), \mathbb{R}_{+}\right)$; 
$\left(H_{11}\right)$ the function $\phi \in C\left(\left[\beta t_{0}, t_{0}\right], \mathbb{R}_{+}\right)$with $\max _{s \in\left[\beta t_{0}, t_{0}\right]} \phi(s)>0$, where $0<\beta<1$;

$\left(H_{12}\right)$ the function $u \in C\left(\left[\beta t_{0}, T\right), \mathbb{R}_{+}\right)$with

$u(t) \leq r(t)+\int_{t_{0}}^{t}(t-s)^{\alpha-1} p(s) \max _{\xi \in[\beta s, s]} u(\xi) d s, \quad t \in\left[t_{0}, T\right)$,

$$
u(t) \leq \phi(t), \quad t \in\left[\beta t_{0}, t_{0}\right]
$$

where $\alpha>0$.

Then the following assertions hold.

$\left(R_{1}\right)$ Suppose $\alpha>1 / 2$; then

$$
\begin{array}{r}
u(t) \leq e^{t}\left[c_{1} r^{2}(t)+h_{1}(t) \exp \left(K_{1} \int_{t_{0}}^{t} p^{2}(s) d s\right)\right]^{1 / 2} \\
t \in\left[t_{0}, T\right)
\end{array}
$$

where

$$
\begin{gathered}
c_{1}=\max \left\{2 e^{-2 t_{0}}, e^{-2 \beta t_{0}}\right\}, \\
K_{1}=\frac{\Gamma(2 \alpha-1)}{4^{\alpha-1}},
\end{gathered}
$$

$h_{1}(t)=c_{1} \max _{s \in\left[\beta t_{0}, t_{0}\right]} \phi^{2}(s)+c_{1} K_{1} \int_{t_{0}}^{t} p^{2}(s) \max _{\xi \in[\beta s, s]} m_{1}^{2}(\xi) d s$,

$$
t \in\left[t_{0}, T\right),
$$

with

$$
m_{1}(t)= \begin{cases}r(t), & t \in\left[t_{0}, T\right) \\ \phi(t), & t \in\left[\beta t_{0}, t_{0}\right]\end{cases}
$$

Moreover, if $r \in C\left(\left[t_{0}, T\right),(0, \infty)\right)$ is a nondecreasing function, then

$$
\begin{array}{r}
u(t) \leq \sqrt{c_{1} N_{1}} r(t) \exp \left(t+\frac{1}{2} K_{1} \int_{t_{0}}^{t} p^{2}(s) d s\right), \\
t \in\left[t_{0}, T\right),
\end{array}
$$

where

$$
N_{1}=\max \left\{1, \frac{\max _{s \in\left[\beta t_{0}, t_{0}\right]} \phi^{2}(s)}{r^{2}\left(t_{0}\right)}\right\} .
$$

$\left(R_{2}\right)$ Suppose $0<\alpha \leq 1 / 2$; then

$$
\begin{array}{r}
u(t) \leq e^{t}\left[c_{2} r^{b}(t)+h_{2}(t) \exp \left(2^{b-1} K_{2}^{b} \int_{t_{0}}^{t} p^{b}(s) d s\right)\right]^{1 / b}, \\
t \in\left[t_{0}, T\right),
\end{array}
$$

where

$$
\begin{gathered}
a=\alpha+1, \\
b=1+\frac{1}{\alpha}, \\
c_{2}=\max \left\{2^{b-1} e^{-b t_{0}}, e^{-b \beta t_{0}}\right\}, \\
K_{2}=\left(\frac{\Gamma\left(\alpha^{2}\right)}{a^{\alpha^{2}}}\right)^{1 / a}, \\
c_{2 \in\left[\beta t_{0}, t_{0}\right]} \phi^{b}(s) \\
+2^{b-1} c_{2} K_{2}^{b} \int_{t_{0}}^{t} p^{b}(s) \max _{\xi \in[\beta s, s]} m_{1}^{b}(\xi) d s, \\
t \in\left[t_{0}, T\right) .
\end{gathered}
$$$$
h_{2}(t)=c_{2} \max _{s \in\left[\beta t_{0}, t_{0}\right]} \phi^{b}(s)
$$

Moreover, if $r \in C\left(\left[t_{0}, T\right),(0, \infty)\right)$ is a nondecreasing function, then

$$
\begin{array}{r}
u(t) \leq\left(c_{2} N_{2}\right)^{1 / b} r(t) \exp \left(t+\frac{2^{b-1}}{b} K_{2}^{b} \int_{t_{0}}^{t} p^{b}(s) d s\right) \\
t \in\left[t_{0}, T\right)
\end{array}
$$

where

$$
N_{2}=\max \left\{1, \frac{\max _{s \in\left[\beta t_{0}, t_{0}\right]} \phi^{b}(s)}{r^{b}\left(t_{0}\right)}\right\}
$$

Proof. Consider $\left(R_{1}\right) \alpha>1 / 2$. Using the Cauchy-Schwarz inequality with (13), we get for $t \in\left[t_{0}, T\right)$

$$
\begin{aligned}
u(t) \leq & r(t)+\int_{t_{0}}^{t}(t-s)^{\alpha-1} e^{s} p(s) e^{-s} \max _{\xi \in[\beta s, s]} u(\xi) d s \\
\leq & r(t)+\left[\int_{t_{0}}^{t}(t-s)^{2 \alpha-2} e^{2 s} d s\right]^{1 / 2} \\
& \times\left[\int_{t_{0}}^{t} p^{2}(s) e^{-2 s}\left(\max _{\xi \in[\beta s, s]} u(\xi)\right)^{2} d s\right]^{1 / 2} .
\end{aligned}
$$

The first integral of (30) implies the estimate

$$
\begin{aligned}
\int_{t_{0}}^{t}(t-s)^{2 \alpha-2} e^{2 s} d s & =\int_{0}^{t-t_{0}} \tau^{2 \alpha-2} e^{2(t-\tau)} d \tau \\
& \leq e^{2 t} \int_{0}^{t} \tau^{2 \alpha-2} e^{-2 \tau} d \tau \\
& =\frac{2 e^{2 t}}{4^{\alpha}} \int_{0}^{2 t} \sigma^{2 \alpha-2} e^{-\sigma} d \sigma \\
& <\frac{2 e^{2 t}}{4^{\alpha}} \Gamma(2 \alpha-1)
\end{aligned}
$$


Therefore, from (30) and (31), we obtain

$$
\begin{aligned}
u(t) \leq & r(t)+\left[\frac{2 e^{2 t}}{4^{\alpha}} \Gamma(2 \alpha-1)\right]^{1 / 2} \\
& \times\left[\int_{t_{0}}^{t} p^{2}(s) e^{-2 s}\left(\max _{\xi \in[\beta s, s]} u(\xi)\right)^{2} d s\right]^{1 / 2} .
\end{aligned}
$$

Applying Lemma 4 with $n=2, \sigma=2$, we get

$$
\begin{aligned}
& u^{2}(t) \leq 2 r^{2}(t) \\
&+\frac{e^{2 t}}{4^{\alpha-1}} \Gamma(2 \alpha-1) \int_{t_{0}}^{t} p^{2}(s) e^{-2 s}\left(\max _{\xi \in[\beta s, s]} u(\xi)\right)^{2} d s, \\
& t \in\left[t_{0}, T\right) .
\end{aligned}
$$

Now, taking $v(t)=\left[e^{-t} u(t)\right]^{2}$, we have

$$
\begin{aligned}
& v(t) \leq 2 e^{-2 t} r^{2}(t)+\frac{\Gamma(2 \alpha-1)}{4^{\alpha-1}} \\
& \times \int_{t_{0}}^{t} p^{2}(s) e^{-2 s}\left(\max _{\xi \in[\beta s, s]} u(\xi)\right)^{2} d s \\
& \leq 2 e^{-2 t_{0}} r^{2}(t)+\frac{\Gamma(2 \alpha-1)}{4^{\alpha-1}} \\
& \times \int_{t_{0}}^{t} p^{2}(s) \max _{\xi \in[\beta s, s]}\left(e^{-2 \xi} u^{2}(\xi)\right) d s \\
& \leq c_{1} r^{2}(t)+K_{1} \int_{t_{0}}^{t} p^{2}(s) \max _{\xi \in[\beta s, s]} v(\xi) d s, \\
& t \in\left[t_{0}, T\right),
\end{aligned}
$$

and, for $t \in\left[\beta t_{0}, t_{0}\right]$,

$$
v(t) \leq e^{-2 t} \phi^{2}(t) \leq e^{-2 \beta t_{0}} \phi^{2}(t) \leq c_{1} \phi^{2}(t),
$$

where $c_{1}$ and $K_{1}$ are defined by (16) and (17), respectively.

Applying Corollary 2 for (34) and (35), we obtain

$$
\begin{array}{r}
v(t) \leq c_{1} r^{2}(t)+h_{1}(t) \exp \left(K_{1} \int_{t_{0}}^{t} p^{2}(s) d s\right), \\
t \in\left[t_{0}, T\right),
\end{array}
$$

where $h_{1}$ is defined by (18). Therefore, we get the required inequality in (15).

Moreover, if $r \in C\left(\left[t_{0}, T\right),(0, \infty)\right)$ is a nondecreasing function, then, by applying Lemma 3 for (34) and (35), we obtain the estimate

$$
v(t) \leq c_{1} N_{1} r^{2}(t) \exp \left(K_{1} \int_{t_{0}}^{t} p^{2}(s) d s\right), \quad t \in\left[t_{0}, T\right),
$$

where $N_{1}$ is defined by (21). Thus, we get the desired inequality in (20). This completes the proof of the first part.
Consider $\left(R_{2}\right) 0<\alpha \leq 1 / 2$. Let $a, b$ be defined by (23) and (24), respectively. It is obvious that $(1 / a)+(1 / b)=1$. Using the Hölder inequality in (13), for $t \in\left[t_{0}, T\right)$, we have

$$
\begin{aligned}
u(t) \leq & r(t)+\int_{t_{0}}^{t}(t-s)^{\alpha-1} e^{s} p(s) e^{-s} \max _{\xi \in[\beta s, s]} u(\xi) d s \\
\leq & r(t)+\left[\int_{t_{0}}^{t}(t-s)^{a(\alpha-1)} e^{a s} d s\right]^{1 / a} \\
& \times\left[\int_{t_{0}}^{t} p^{b}(s) e^{-b s}\left(\max _{\xi \in[\beta s, s]} u(\xi)\right)^{b} d s\right]^{1 / b} .
\end{aligned}
$$

Repeating the process to get (31), the first integral of (38) implies the estimate

$$
\int_{t_{0}}^{t}(t-s)^{a(\alpha-1)} e^{a s} d s<\frac{e^{a t}}{a^{1-a(1-\alpha)}} \Gamma(1-a(1-\alpha)) .
$$

Obviously, $1-a(1-\alpha)=\alpha^{2}>0$ and $\Gamma(1-a(1-\alpha)) \in \mathbb{R}$. From (38) and (39), it follows that

$$
u(t) \leq r(t)+K_{2} e^{t}\left[\int_{t_{0}}^{t} p^{b}(s) e^{-b s}\left(\max _{\xi \in[\beta s, s]} u(\xi)\right)^{b} d s\right]^{1 / b}
$$

where $K_{2}$ is defined by (26). Applying Lemma 4 with $n=2$, $\sigma=b$, we have

$u^{b}(t)$

$$
\begin{array}{r}
\leq 2^{b-1}\left[r^{b}(t)+K_{2}^{b} e^{b t} \int_{t_{0}}^{t} p^{b}(s) e^{-b s}\left(\max _{\xi \in[\beta s, s]} u(\xi)\right)^{b} d s\right], \\
t \in\left[t_{0}, T\right) .
\end{array}
$$

By setting $v(t)=\left[e^{-t} u(t)\right]^{b}$, we get

$$
\begin{array}{r}
v(t) \leq c_{2} r^{b}(t)+2^{b-1} K_{2}^{b} \int_{t_{0}}^{t} p^{b}(s) \max _{\xi \in[\beta s, s]} v(\xi) d s, \\
t \in\left[t_{0}, T\right),
\end{array}
$$

and, for $t \in\left[\beta t_{0}, t_{0}\right]$,

$$
v(t) \leq e^{-b \beta t_{0}} \phi^{b}(t) \leq c_{2} \phi^{b}(t),
$$

where $c_{2}$ is defined by (25). Consequently, applying Corollary 2 with (42) and (43), we have

$$
\begin{array}{r}
v(t) \leq c_{2} r^{b}(t)+h_{2}(t) \exp \left(2{ }^{b-1} K_{2}^{b} \int_{t_{0}}^{t} p^{b}(s) d s\right), \\
t \in\left[t_{0}, T\right),
\end{array}
$$

where $h_{2}$ is defined by (27). Therefore, the desired inequality (22) is established. 
Furthermore, if $r \in C\left(\left[t_{0}, T\right),(0, \infty)\right)$ is a nondecreasing function, then by applying Lemma 3 for (42) and (43) we deduce that

$$
\begin{array}{r}
v(t) \leq c_{2} N_{2} r^{b}(t) \exp \left(2^{b-1} K_{2}^{b} \int_{t_{0}}^{t} p^{b}(s) d s\right), \\
t \in\left[t_{0}, T\right) .
\end{array}
$$

Thus, inequality (28) is proved. This completes the proof.

Theorem 6. Assume that

$\left(H_{13}\right)$ the conditions $\left(H_{10}\right),\left(H_{11}\right)$ of Theorem 5 are satisfied; $\left(H_{14}\right)$ the function $q \in C\left(\left[t_{0}, T\right), \mathbb{R}_{+}\right)$;

$\left(H_{15}\right)$ the function $u \in C\left(\left[\beta t_{0}, T\right), \mathbb{R}_{+}\right)$with

$u(t)$

$$
\begin{array}{r}
\leq r(t)+\int_{t_{0}}^{t}(t-s)^{\alpha-1}\left[p(s) u(s)+q(s) \max _{\xi \in[\beta s, s]} u(\xi)\right] d s, \\
t \in\left[t_{0}, T\right), \\
u(t) \leq \phi(t), \quad t \in\left[\beta t_{0}, t_{0}\right],
\end{array}
$$

where $\alpha>0$.

Then the following assertions hold.

$\left(R_{3}\right)$ Suppose $\alpha>1 / 2$; then

$u(t)$

$$
\begin{array}{r}
\leq e^{t}\left[c_{3} r^{2}(t)+h_{3}(t) \exp \left(K_{3} \int_{t_{0}}^{t}\left[p^{2}(s)+q^{2}(s)\right] d s\right)\right]^{1 / 2}, \\
t \in\left[t_{0}, T\right),
\end{array}
$$

where

$$
\begin{gathered}
c_{3}=\max \left\{3 e^{-2 t_{0}}, e^{-2 \beta t_{0}}\right\}, \\
K_{3}=\frac{6}{4^{\alpha}} \Gamma(2 \alpha-1), \\
h_{3}(t)=c_{3} \max _{s \in\left[\beta t_{0}, t_{0}\right]} \phi^{2}(s)+c_{3} K_{3} \\
\times \int_{t_{0}}^{t}\left[p^{2}(s) r^{2}(s)+q^{2}(s) \max _{\xi \in[\beta s, s]} m_{1}^{2}(\xi)\right] d s, \\
t \in\left[t_{0}, T\right),
\end{gathered}
$$

with $m_{1}$ being defined by (19).
Furthermore, if $r \in C\left(\left[t_{0}, T\right),(0, \infty)\right)$ is a nondecreasing function, then

$$
u(t) \leq \sqrt{c_{3} N_{1}} r(t) \exp \left(t+\frac{1}{2} K_{3} \int_{t_{0}}^{t}\left[p^{2}(s)+q^{2}(s)\right] d s\right),
$$

$$
t \in\left[t_{0}, T\right)
$$

where $N_{1}$ is defined by (21).

$\left(R_{4}\right)$ Suppose $0<\alpha \leq 1 / 2$; then

$u(t) \leq e^{t}\left[c_{4} r^{b}(t)\right.$

$$
\begin{array}{r}
\left.+h_{4}(t) \exp \left(3^{b-1} K_{2}^{b} \int_{t_{0}}^{t}\left[p^{b}(s)+q^{b}(s)\right] d s\right)\right]^{1 / b}, \\
t \in\left[t_{0}, T\right),
\end{array}
$$

where $a, b$, and $K_{2}$ are defined by (23), (24), and (26), respectively,

$$
\begin{gathered}
c_{4}=\max \left\{3^{b-1} e^{-b t_{0}}, e^{-b \beta t_{0}}\right\}, \\
h_{4}(t)=c_{4} \max _{s \in\left[\beta t_{0}, t_{0}\right]} \phi^{b}(s)+c_{4} 3^{b-1} K_{2}^{b} \\
\times \int_{t_{0}}^{t}\left[p^{b}(s) r^{b}(s)+q^{b}(s) \max _{\xi \in[\beta s, s]} m_{1}^{b}(\xi)\right] d s, \\
t \in\left[t_{0}, T\right) .
\end{gathered}
$$

Furthermore, if $r \in C\left(\left[t_{0}, T\right),(0, \infty)\right)$ is a nondecreasing function, then

$u(t)$

$$
\leq\left(c_{4} N_{2}\right)^{1 / b} r(t) \exp \left(t+\frac{3^{b-1}}{b} K_{2}^{b} \int_{t_{0}}^{t}\left[p^{b}(s)+q^{b}(s)\right] d s\right),
$$

$$
t \in\left[t_{0}, T\right),
$$

where $\mathrm{N}_{2}$ is defined by (29).

Proof. Consider $\left(R_{3}\right) \alpha>1 / 2$. By using the Cauchy-Schwarz inequality in (46), for $t \in\left[t_{0}, T\right)$, we have

$$
\begin{aligned}
u(t) \leq & r(t)+\int_{t_{0}}^{t}(t-s)^{\alpha-1} e^{s} p(s) e^{-s} u(s) d s \\
& +\int_{t_{0}}^{t}(t-s)^{\alpha-1} e^{s} q(s) e^{-s} \max _{\xi \in[\beta s, s]} u(\xi) d s \\
\leq & r(t)+\left[\int_{t_{0}}^{t}(t-s)^{2 \alpha-2} e^{2 s} d s\right]^{1 / 2}
\end{aligned}
$$




$$
\begin{aligned}
& \times\left[\int_{t_{0}}^{t} p^{2}(s) e^{-2 s} u^{2}(s) d s\right]^{1 / 2} \\
& +\left[\int_{t_{0}}^{t}(t-s)^{2 \alpha-2} e^{2 s} d s\right]^{1 / 2} \\
& \times\left[\int_{t_{0}}^{t} q^{2}(s) e^{-2 s}\left(\max _{\xi \in[\beta s, s]} u(\xi)\right)^{2} d s\right]^{1 / 2} \\
& \leq r(t)+\left[\frac{2 e^{2 t}}{4^{\alpha}} \Gamma(2 \alpha-1)\right]^{1 / 2} \\
& \times\left\{\left[\int_{t_{0}}^{t} p^{2}(s) e^{-2 s} u^{2}(s) d s\right]^{1 / 2}\right. \\
& \left.+\left[\int_{t_{0}}^{t} q^{2}(s) e^{-2 s}\left(\max _{\xi \in[\beta s, s]} u(\xi)\right)^{2} d s\right]^{1 / 2}\right\} .
\end{aligned}
$$

Applying Lemma 4 with $n=3, \sigma=2$, we get

$$
\begin{aligned}
& u^{2}(t) \leq 3 r^{2}(t)+\frac{6 e^{2 t}}{4^{\alpha}} \Gamma(2 \alpha-1) \\
& \times\left[\int_{t_{0}}^{t} p^{2}(s) e^{-2 s} u^{2}(s) d s\right. \\
&\left.+\int_{t_{0}}^{t} q^{2}(s) e^{-2 s}\left(\max _{\xi \in[\beta s, s]} u(\xi)\right)^{2} d s\right], \\
& t \in\left[t_{0}, T\right) .
\end{aligned}
$$

$$
\begin{gathered}
+K_{3} \int_{t_{0}}^{t} q^{2}(s) \max _{\xi \in[\beta s, s]} v(\xi) d s, \quad t \in\left[t_{0}, T\right), \\
v(t) \leq c_{3} \phi^{2}(t), \quad t \in\left[\beta t_{0}, t_{0}\right],
\end{gathered}
$$

where $c_{3}$ and $K_{3}$ are defined by (49) and (50), respectively. Using Corollary 2 for (59) and (60), it follows that

$$
\begin{array}{r}
v(t) \leq c_{3} r^{2}(t)+h_{3}(t) \exp \left(K_{3} \int_{t_{0}}^{t}\left[p^{2}(s)+q^{2}(s)\right] d s\right), \\
t \in\left[t_{0}, T\right),
\end{array}
$$

where $h_{3}$ is defined by (51). Thus, we get the result in (48).

If $r \in C\left(\left[t_{0}, T\right),(0, \infty)\right)$ is a nondecreasing function, then Lemma 3 with (59) and (60) implies the estimate

$$
\begin{array}{r}
v(t) \leq c_{3} N_{1} r^{2}(t) \exp \left(K_{3} \int_{t_{0}}^{t}\left[p^{2}(s)+q^{2}(s)\right] d s\right), \\
t \in\left[t_{0}, T\right),
\end{array}
$$

where $N_{1}$ is defined by (21). Thus, the required inequality (52) is established. This completes the proof of the first part.

Consider $\left(R_{4}\right) 0<\alpha \leq 1 / 2$. Let $a, b$ be defined by (23) and (24), respectively. Applying the Hölder inequality in (46), we have that for $t \in\left[t_{0}, T\right)$

$$
\begin{aligned}
u(t) \leq & r(t)+\left[\int_{t_{0}}^{t}(t-s)^{a(\alpha-1)} e^{a s} d s\right]^{1 / a} \\
& \times\left[\int_{t_{0}}^{t} p^{b}(s) e^{-b s} u^{b}(s) d s\right]^{1 / b} \\
& +\left[\int_{t_{0}}^{t}(t-s)^{a(\alpha-1)} e^{a s} d s\right]^{1 / a} \\
& \times\left[\int_{t_{0}}^{t} q^{b}(s) e^{-b s}\left(\max _{\xi \in[\beta s, s]} u(\xi)\right)^{b} d s\right]^{1 / b} \\
\leq & r(t)+K_{2} e^{t}\left\{\left[\int_{t_{0}}^{t} p^{b}(s) e^{-b s} u^{b}(s) d s\right]^{1 / b}\right. \\
& \left.+\left[\int_{t_{0}}^{t} q^{b}(s) e^{-b s}\left(\max _{\xi \in[\beta s, s]} u(\xi)\right)^{b} d s\right]^{1 / b}\right\}
\end{aligned}
$$

where $K_{2}$ is defined by (26). By using Lemma 4 with $n=3$, $\sigma=b$, we obtain the estimate

$$
\begin{aligned}
& u^{b}(t) \leq 3^{b-1} r^{b}(t)+3^{b-1} K_{2}^{b} e^{b t} \\
& \times\left[\int_{t_{0}}^{t} p^{b}(s) e^{-b s} u^{b}(s) d s\right. \\
&\left.+\int_{t_{0}}^{t} q^{b}(s) e^{-b s}\left(\max _{\xi \in[\beta s, s]} u(\xi)\right)^{b} d s\right], \\
& t \in\left[t_{0}, T\right) .
\end{aligned}
$$

Substituting $v(t)=\left[e^{-t} u(t)\right]^{b}$, we get

$$
\begin{aligned}
v(t) \leq & c_{4} r^{b}(t)+3^{b-1} K_{2}^{b} \\
\times & {\left[\int_{t_{0}}^{t} p^{b}(s) v(s) d s\right.} \\
& \left.+\int_{t_{0}}^{t} q^{b}(s) \max _{\xi \in[\beta s, s]} v(\xi) d s\right], \quad t \in\left[t_{0}, T\right),
\end{aligned}
$$


and, for $t \in\left[\beta t_{0}, t_{0}\right]$,

$$
v(t) \leq c_{4} \phi^{b}(t)
$$

where $c_{4}$ is defined by (54). An application of Corollary 2 to (65) and (66) gives

$$
\begin{array}{r}
v(t) \leq c_{4} r^{b}(t)+h_{4}(t) \\
\times \exp \left(3^{b-1} K_{2}^{b} \int_{t_{0}}^{t}\left[p^{b}(s)+q^{b}(s)\right] d s\right), \\
t \in\left[t_{0}, T\right),
\end{array}
$$

where $h_{4}$ is defined by (55). Therefore, we deduce inequality (53).

As a special case, if $r \in C\left(\left[t_{0}, T\right),(0, \infty)\right)$ is a nondecreasing function, then, by Lemma 3 with (65) and (66), we get

$$
\begin{array}{rl}
v(t) \leq & c_{4} N_{2} r^{b}(t) \\
& \times \exp \left(3^{b-1} K_{2}^{b} \int_{t_{0}}^{t}\left[p^{b}(s)+q^{b}(s)\right] d s\right), \\
t & t \in\left[t_{0}, T\right) .
\end{array}
$$

Therefore, the desired inequality (56) is established. This completes the proof of Theorem 6 .

\section{Applications to Fractional Differential Equations with "Maxima"}

In this section, we apply our results to demonstrate the bound of solutions and the dependence of solutions on the orders with initial conditions for Caputo fractional differential equations with "maxima." We consider the following fractional differential equations (FDEs) with "maxima"

$$
D^{\alpha} x(t)=f\left(t, x(t), \max _{s \in[\beta t, t]} x(s)\right), \quad t \in I=\left[t_{0}, T\right),
$$

and initial condition

$$
x(t)=\phi(t), \quad t \in\left[\beta t_{0}, t_{0}\right],
$$

where $D^{\alpha}$ represents the Caputo fractional derivative of order $\alpha(\alpha>0), f \in C(I \times \mathbb{R} \times \mathbb{R}, \mathbb{R}), \phi$ is a given continuously differentiable function on $\left[\beta t_{0}, t_{0}\right]$ up to order $n(n=-[-\alpha])$, and $0<\beta<1$. We denote $\phi^{(k)}\left(t_{0}\right)=\rho_{k}, k=0,1,2, \ldots, n-1$. For more details on fractional differential equations, see [23, 24].

Theorem 7. Assume that

$\left(H_{16}\right)$ there exist functions $y, z \in C\left(I, \mathbb{R}_{+}\right)$such that, for $t \in I$, $u_{1}, u_{2} \in \mathbb{R}$,

$$
\left|f\left(t, u_{1}, u_{2}\right)\right| \leq y(t)\left|u_{1}\right|+z(t)\left|u_{2}\right| .
$$

If $x$ is solution of the initial value problem (69)-(70), then the following estimates hold.
$\left(R_{5}\right)$ Suppose $1 / 2<\alpha \leq 1$. Then

$$
\begin{array}{r}
|x(t)| \leq \sqrt{c_{3}} M \exp \left(t+\frac{K_{3}}{2 \Gamma^{2}(\alpha)} \int_{t_{0}}^{t}\left[y^{2}(s)+z^{2}(s)\right] d s\right), \\
t \in I .
\end{array}
$$

$\left(R_{6}\right)$ Suppose $0<\alpha \leq 1 / 2$. Then

$$
|x(t)| \leq\left(c_{4}\right)^{1 / b} M \exp \left(t+\frac{3^{b-1} K_{2}^{b}}{b \Gamma^{b}(\alpha)} \int_{t_{0}}^{t}\left[y^{b}(s)+z^{b}(s)\right] d s\right),
$$

$\left(R_{7}\right)$ Suppose $\alpha>1$. Then

$$
\begin{aligned}
|x(t)| \leq & \sqrt{c_{3}}\left(M+\sum_{j=1}^{n-1} \frac{\left|\rho_{j}\right|}{j !}\left(t-t_{0}\right)^{j}\right) \\
& \times \exp \left(t+\frac{K_{3}}{2 \Gamma^{2}(\alpha)} \int_{t_{0}}^{t}\left[y^{2}(s)+z^{2}(s)\right] d s\right),
\end{aligned}
$$

$t \in I$,

where

$$
M=\max _{t \in\left[\beta t_{0}, t_{0}\right]}|\phi(t)|>0
$$

and $b, c_{3}, c_{4}, K_{2}$, and $K_{3}$ are defined as in Theorems 5 and 6 .

Proof. The solution $x$ of the initial value problem (69)-(70) satisfies the following equations (see [23]):

$$
\begin{gathered}
x(t)=\sum_{j=0}^{n-1} \frac{\rho_{j}}{j !}\left(t-t_{0}\right)^{j}+\frac{1}{\Gamma(\alpha)} \\
\quad \times \int_{t_{0}}^{t}(t-s)^{\alpha-1} f\left(s, x(s), \max _{\xi \in[\beta s, s]} x(\xi)\right) d s, \\
x(t)=\phi(t), \quad t \in\left[\beta t_{0}, t_{0}\right] .
\end{gathered}
$$

For $0<\alpha \leq 1$, by using the assumption $\left(H_{16}\right)$, it follows that $|x(t)|$

$$
\begin{array}{rl}
\leq & M+\frac{1}{\Gamma(\alpha)} \\
& \times \int_{t_{0}}^{t}(t-s)^{\alpha-1}\left(y(s)|x(s)|+z(s) \max _{\xi \in[\beta s, s]}|x(\xi)|\right) d s, \\
t & t \in I,
\end{array}
$$$$
|x(t)| \leq M, \quad t \in\left[\beta t_{0}, t_{0}\right] .
$$ 
Hence, Theorem 6 yields the estimate inequalities (72) and (73).

For $\alpha>1$, by using the assumption $\left(H_{16}\right)$ in $(76)$, we have

$$
\begin{aligned}
|x(t)| \leq & M+\sum_{j=1}^{n-1} \frac{\left|\rho_{j}\right|}{j !}\left(t-t_{0}\right)^{j} \\
& +\frac{1}{\Gamma(\alpha)} \int_{t_{0}}^{t}(t-s)^{\alpha-1} \\
& \times\left(y(s)|x(s)|+z(s) \max _{\xi \in[\beta s, s]}|x(\xi)|\right) d s,
\end{aligned}
$$$$
t \in I
$$$$
|x(t)| \leq M, \quad t \in\left[\beta t_{0}, t_{0}\right] .
$$

Since $\sum_{j=1}^{n-1}\left(\left|\rho_{j}\right| / j !\right)\left(t-t_{0}\right)^{j}$ is a nondecreasing function, Theorem 6 yields the estimate inequality (74). This completes the proof.

Theorem 8. Let $\alpha>0$ and $\delta>0$ such that $0 \leq n-1<\alpha-\delta<$ $\alpha \leq n$. Also let $f: I \times \mathbb{R} \times \mathbb{R} \rightarrow \mathbb{R}$ be a continuous function satisfying the following assumption:

$$
\begin{aligned}
& \left(H_{17}\right) \text { there exist constants } L_{1}, L_{2}>0 \text { such that } \mid f\left(t, u_{1}, u_{2}\right)- \\
& f\left(t, v_{1}, v_{2}\right)\left|\leq L_{1}\right| u_{1}-v_{1}\left|+L_{2}\right| u_{2}-v_{2} \mid, \text { for each } t \in I \\
& \text { and } u_{1}, u_{2}, v_{1}, v_{2} \in \mathbb{R} .
\end{aligned}
$$

If $x$ and $y$ are the solutions of the initial value problem (69)(70) and

$$
D^{\alpha-\delta} y(t)=f\left(t, y(t), \max _{s \in[\beta t, t]} y(s)\right), \quad t \in I,
$$

with initial condition

$$
y(t)=\bar{\phi}(t), \quad t \in\left[\beta t_{0}, t_{0}\right]
$$

respectively, where $\bar{\phi}$ is a given continuous function on $\left[\beta t_{0}, t_{0}\right]$ such that $\phi(t) \neq \bar{\phi}(t)$ for all $t \in\left[\beta t_{0}, t_{0}\right)$ up to order $n(n=$ $-[-(\alpha-\delta)])$. we denote $\bar{\phi}^{(k)}\left(t_{0}\right)=\bar{\rho}_{k}, k=0,1,2, \ldots, n-1$. Then the following estimates hold for $t_{0}<t \leq h<T$.

$$
\left(R_{8}\right) \text { Suppose } \alpha-\delta>1 / 2 \text {. Then for } t \in I
$$

$$
\begin{aligned}
& |y(t)-x(t)| \\
& \leq e^{t}\left[c_{5} A^{2}(t)+h_{5}(t)\right. \\
& \left.\quad \times \exp \left(\frac{6 \Gamma(2 \alpha-2 \delta-1)\left(L_{1}^{2}+L_{2}^{2}\right)\left(t-t_{0}\right)}{4^{\alpha-\delta} \Gamma^{2}(\alpha)}\right)\right]^{1 / 2} .
\end{aligned}
$$

$$
\left(R_{9}\right) \text { Suppose } 0<\alpha-\delta \leq 1 / 2 \text {. Then for } t \in I
$$

$$
\begin{aligned}
& |y(t)-x(t)| \\
& \leq e^{t}\left[c_{6} A^{b}(t)+h_{6}(t)\right. \\
& \left.\quad \times \exp \left(\frac{\left[3 \Gamma\left((\alpha-\delta)^{2}\right)\right]^{1 /(\alpha-\delta)}\left(L_{1}^{b}+L_{2}^{b}\right)\left(t-t_{0}\right)}{(\alpha-\delta+1)^{\alpha-\delta} \Gamma^{b}(\alpha)}\right)\right]^{1 / b},
\end{aligned}
$$

where

$$
\begin{aligned}
& A(t)=\left|\sum_{j=0}^{n-1} \frac{\left(\bar{\rho}_{j}-\rho_{j}\right)\left(t-t_{0}\right)^{j}}{j !}\right| \\
& +\left|\frac{\left(t-t_{0}\right)^{\alpha-\delta}}{\Gamma(\alpha-\delta+1)}-\frac{\left(t-t_{0}\right)^{\alpha-\delta}}{(\alpha-\delta) \Gamma(\alpha)}\right|\|f\| \\
& +\left|\frac{\left(t-t_{0}\right)^{\alpha-\delta}}{(\alpha-\delta) \Gamma(\alpha)}-\frac{\left(t-t_{0}\right)^{\alpha}}{\Gamma(\alpha+1)}\right|\|f\|, \\
& \|f\|=\sup _{t_{0} \leq t \leq h}\left|f\left(t, y(t), \max _{s \in[\beta t, t]} y(s)\right)\right| \text {, } \\
& b=1+\frac{1}{\alpha-\delta}, \\
& c_{5}=\max \left\{3 e^{-2 t_{0}}, e^{-2 \beta t_{0}}\right\} \text {, } \\
& c_{6}=\max \left\{3^{1 /(\alpha-\delta)} e^{-b t_{0}}, e^{-b \beta t_{0}}\right\}, \\
& h_{5}(t)=c_{5} \max _{s \in\left[\beta t_{0}, t_{0}\right]}|\bar{\phi}(s)-\phi(s)|^{2} \\
& +\frac{6 c_{5} \Gamma(2 \alpha-2 \delta-1)}{4^{\alpha-\delta} \Gamma^{2}(\alpha)} \\
& \times \int_{t_{0}}^{t}\left(L_{1}^{2} A^{2}(s)+L_{2}^{2} \max _{\xi \in[\beta s, s]} m_{2}^{2}(\xi)\right) d s, \\
& h_{6}(t)=c_{6} \max _{s \in\left[\beta t_{0}, t_{0}\right]}|\bar{\phi}(s)-\phi(s)|^{b} \\
& +\frac{c_{6}\left[3 \Gamma\left((\alpha-\delta)^{2}\right)\right]^{1 /(\alpha-\delta)}}{(\alpha-\delta+1)^{\alpha-\delta} \Gamma^{b}(\alpha)} \\
& \times \int_{t_{0}}^{t}\left(L_{1}^{b} A^{b}(s)+L_{2}^{b} \max _{\xi \in[\beta s, s]} m_{2}^{b}(\xi)\right) d s,
\end{aligned}
$$

with

$$
m_{2}(t)= \begin{cases}A(t), & t \in I \\ |\bar{\phi}(t)-\phi(t)|, & t \in\left[\beta t_{0}, t_{0}\right]\end{cases}
$$


Proof. The solutions $x$ and $y$ of the initial value problems (69)-(70) and (80)-(81) satisfy the following equations:

$$
\begin{aligned}
x(t)= & \sum_{j=0}^{n-1} \frac{\rho_{j}}{j !}\left(t-t_{0}\right)^{j}+\frac{1}{\Gamma(\alpha)} \\
& \times \int_{t_{0}}^{t}(t-s)^{\alpha-1} f\left(s, x(s), \max _{\xi \in[\beta s, s]} x(\xi)\right) d s, \\
y(t)= & \sum_{j=0}^{n-1} \frac{\bar{\rho}_{j}}{j !}\left(t-t_{0}\right)^{j}+\frac{1}{\Gamma(\alpha-\delta)} \\
& \times \int_{t_{0}}^{t}(t-s)^{\alpha-\delta-1} f\left(s, y(s), \max _{\xi \in[\beta s, s]} y(\xi)\right) d s,
\end{aligned}
$$

respectively. So, using the assumption $\left(H_{17}\right)$, it follows that

$$
\begin{aligned}
& |y(t)-x(t)| \\
& \leq\left|\sum_{j=0}^{n-1} \frac{\bar{\rho}_{j}}{j !}\left(t-t_{0}\right)^{j}-\sum_{j=0}^{n-1} \frac{\rho_{j}}{j !}\left(t-t_{0}\right)^{j}\right| \\
& +\mid \frac{1}{\Gamma(\alpha-\delta)} \int_{t_{0}}^{t}(t-s)^{\alpha-\delta-1} \\
& \times f\left(s, y(s), \max _{\xi \in[\beta s, s]} y(\xi)\right) d s \\
& -\frac{1}{\Gamma(\alpha)} \int_{t_{0}}^{t}(t-s)^{\alpha-\delta-1} \\
& \times f\left(s, y(s), \max _{\xi \in[\beta s, s]} y(\xi)\right) d s \\
& +\mid \frac{1}{\Gamma(\alpha)} \int_{t_{0}}^{t}(t-s)^{\alpha-\delta-1} \\
& \times f\left(s, y(s), \max _{\xi \in[\beta s, s]} y(\xi)\right) d s \\
& -\frac{1}{\Gamma(\alpha)} \int_{t_{0}}^{t}(t-s)^{\alpha-\delta-1} \\
& \times f\left(s, x(s), \max _{\xi \in[\beta s, s]} x(\xi)\right) d s \\
& +\mid \frac{1}{\Gamma(\alpha)} \int_{t_{0}}^{t}(t-s)^{\alpha-\delta-1} \\
& \times f\left(s, x(s), \max _{\xi \in[\beta s, s]} x(\xi)\right) d s \\
& -\frac{1}{\Gamma(\alpha)} \int_{t_{0}}^{t}(t-s)^{\alpha-1}
\end{aligned}
$$

$$
\begin{aligned}
& \quad \times f\left(s, x(s) \max _{\xi \in[\beta s, s]} x(\xi)\right) d s \mid \\
& \leq A(t)+\frac{1}{\Gamma(\alpha)} \int_{t_{0}}^{t}(t-s)^{\alpha-\delta-1} \\
& \times\left(L_{1}|y(s)-x(s)|\right. \\
& \left.\quad+L_{2}\left|\max _{\xi \in[\beta s, s]} y(\xi)-\max _{\xi \in[\beta s, s]} x(\xi)\right|\right) d s \\
& \leq A(t)+\frac{1}{\Gamma(\alpha)} \int_{t_{0}}^{t}(t-s)^{\alpha-\delta-1} \\
& \times\left(L_{1}|y(s)-x(s)|\right. \\
& \left.\quad+L_{2} \max _{\xi \in[\beta s, s]}|y(\xi)-x(\xi)|\right) d s,
\end{aligned}
$$

where $A(t)$ is defined by (84) and

$$
|y(t)-x(t)|=|\bar{\phi}(t)-\phi(t)|, \quad t \in\left[\beta t_{0}, t_{0}\right] .
$$

Applying Theorem 6 yields the desired inequalities (82) and (83). This completes the proof.

\section{Conflict of Interests}

The authors declare that there is no conflict of interests regarding the publication of this paper.

\section{Acknowledgments}

This research is supported by the Centre of Excellence in Mathematics, the Commission on Higher Education, Thailand. Sotiris K. Ntouyas is a member of Nonlinear Analysis and Applied Mathematics (NAAM) Research Group at King Abdulaziz University, Jeddah, Saudi Arabia.

\section{References}

[1] B. G. Pachpatte, "On some generalizations of Bellman's lemma," Journal of Mathematical Analysis and Applications, vol. 5, pp. 141-150, 1995.

[2] B. G. Pachpatte, Inequalities for Differential and Integral Equations, Academic Press, New York, NY, USA, 1998.

[3] B. G. Pachpatte, "Explicit bounds on certain integral inequalities," Journal of Mathematical Analysis and Applications, vol. 267, no. 1, pp. 48-61, 2002.

[4] B. G. Pachpatte, Integral and Finite Difference Inequalities and Applications, Elsevier, Amsterdam, The Netherlands, 2006.

[5] O. Lipovan, "A retarded Gronwall-like inequality and its applications," Journal of Mathematical Analysis and Applications, vol. 252, no. 1, pp. 389-401, 2000. 
[6] D. Henry, Geometric Theory of Semilinear Parabolic Equations, Springer, New York, NY, USA, 1981.

[7] M. Medved', "A new approach to an analysis of Henry type integral inequalities and their Bihari type versions," Journal of Mathematical Analysis and Applications, vol. 214, no. 2, pp. 349366, 1997.

[8] M. Medved, "Integral inequalities and global solutions of semilinear evolution equations," Journal of Mathematical Analysis and Applications, vol. 267, no. 2, pp. 643-650, 2002.

[9] H. Ye and J. Gao, "Henry-Gronwall type retarded integral inequalities and their applications to fractional differential equations with delay," Applied Mathematics and Computation, vol. 218, no. 8, pp. 4152-4160, 2011.

[10] Q. Ma and J. Pečarić, "Some new explicit bounds for weakly singular integral inequalities with applications to fractional differential and integral equations," Journal of Mathematical Analysis and Applications, vol. 341, no. 2, pp. 894-905, 2008.

[11] J. Shao and F. Meng, "Gronwall-Bellman type inequalities and their applications to fractional differential equations," Abstract and Applied Analysis, vol. 2013, Article ID 217641, 7 pages, 2013.

[12] D. Qian, Z. Gong, and C. Li, "A generalized Gronwall inequality and its application to fractional differential equations with Hadamard derivatives," in Proceedings of the 3rd Conference on Nonlinear Science and Complexity (NSC '10), pp. 1-4, Cankaya University, 2010.

[13] S. Y. Lin, "Generalized Gronwall inequalities and their applications to fractional differential equations," Journal of Mathematical Analysis and Applications, vol. 2013, article 549, 2013.

[14] R. P. Agarwal, S. Deng, and W. Zhang, "Generalization of a retarded Gronwall-like inequality and its applications," Applied Mathematics and Computation, vol. 165, no. 3, pp. 599-612, 2005.

[15] Z. Denton and A. S. Vatsala, "Fractional integral inequalities and applications," Computers and Mathematics with Applications, vol. 59, no. 3, pp. 1087-1094, 2010.

[16] H. El-Owaidy, A. Ragab, and A. Abdeldaim, "On some new integral inequalities of Growall-Bellman type," Applied Mathematics and Computation, vol. 106, no. 2-3, pp. 289-303, 1999.

[17] H. Ye, Y. Ding, and J. Gao, "The existence of a positive solution of $D^{\alpha}[x(t)-x(0)] x(t) f\left(t, x_{t}\right)$, Positivity, vol. 11, no. 2, pp. 341$350,2007$.

[18] H. Ye, J. Gao, and Y. Ding, "A generalized Gronwall inequality and its application to a fractional differential equation," Journal of Mathematical Analysis and Applications, vol. 328, no. 2, pp. 1075-1081, 2007.

[19] D. Bainov and S. Hristova, Differential Equations with Maxima, Pure and Applied Mathematics, Chapman \& Hall/CRC, New York, NY, USA, 2011.

[20] S. Hristova and K. Stefanova, "Some integral inequalities with maximum of the unknown functions," Advances in Dynamical Systems and Applications, vol. 6, pp. 57-69, 2011.

[21] J. Tariboon, P. Thiramanus, and S. K. Ntouyas, "Dynamic integral inequalities on time scales with 'maxima"' Journal of Inequalities and Applications, vol. 2013, article 564, 2013.

[22] M. Kuczma, An Introduction to the Theory of Functional Equations and Inequalities: Cauchy's Equation and Jensen's Inequality, Birkhäauser, 2009.

[23] A. A. Kilbas, H. M. Srivastava, and J. J. Trujillo, Theory and Applications of Fractional Differential Equations, vol. 204 of North-Holland Mathematics Studies, Elsevier, 2006.

[24] I. Podlubny, Fractional Differential Equations, Academic Press, New York, NY, USA, 1999. 


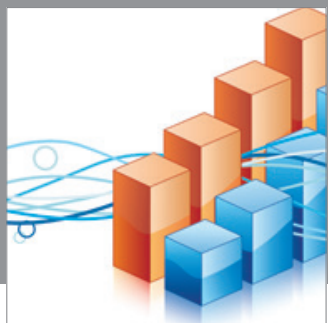

Advances in

Operations Research

mansans

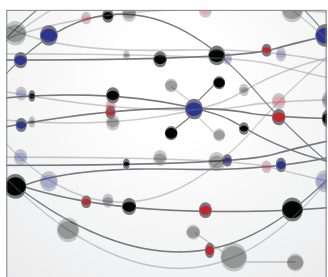

The Scientific World Journal
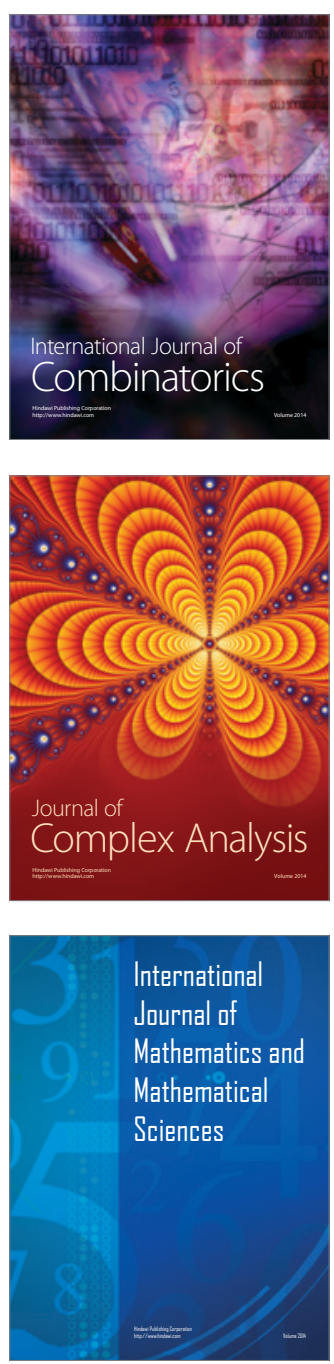
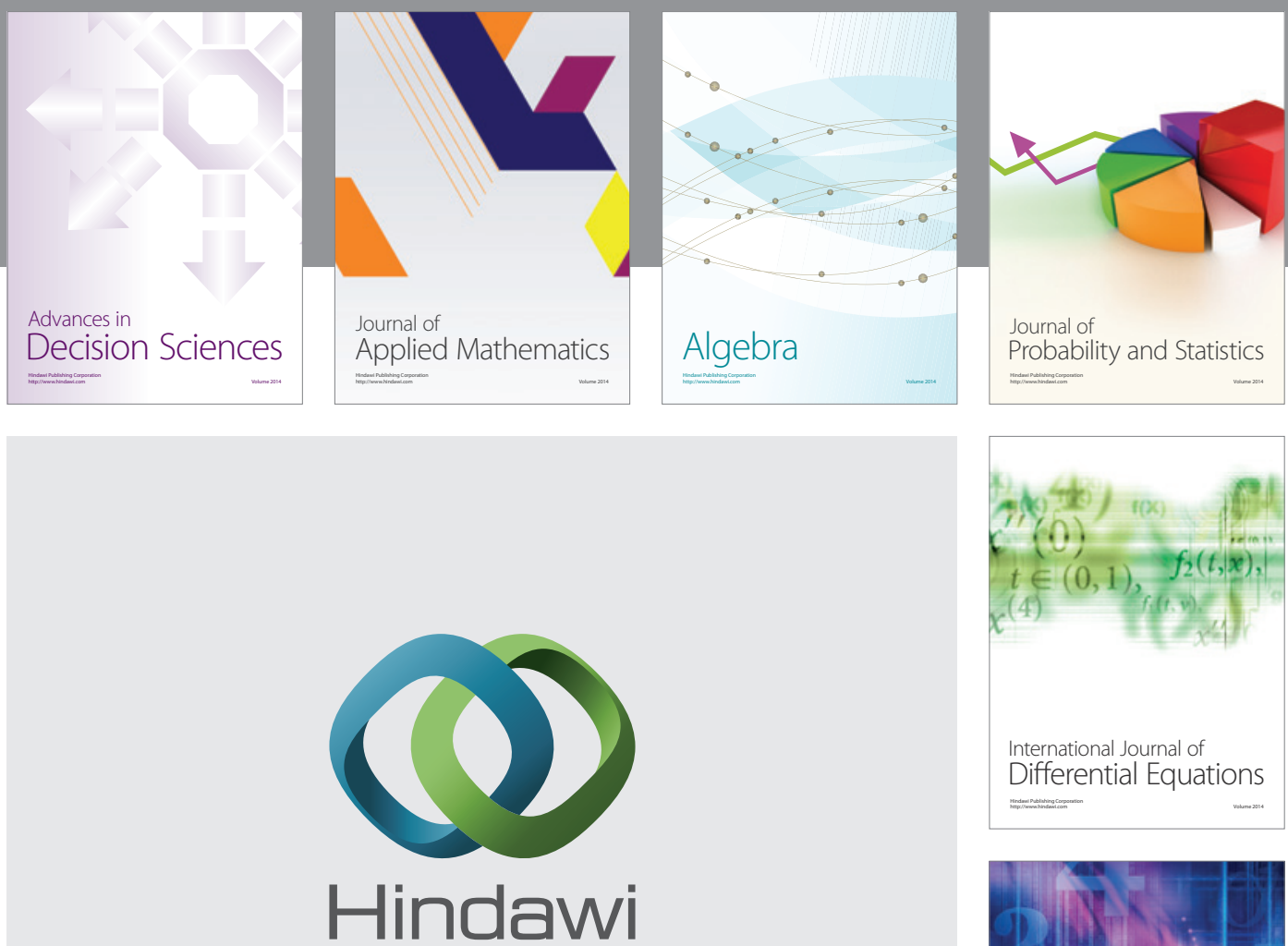

Submit your manuscripts at http://www.hindawi.com
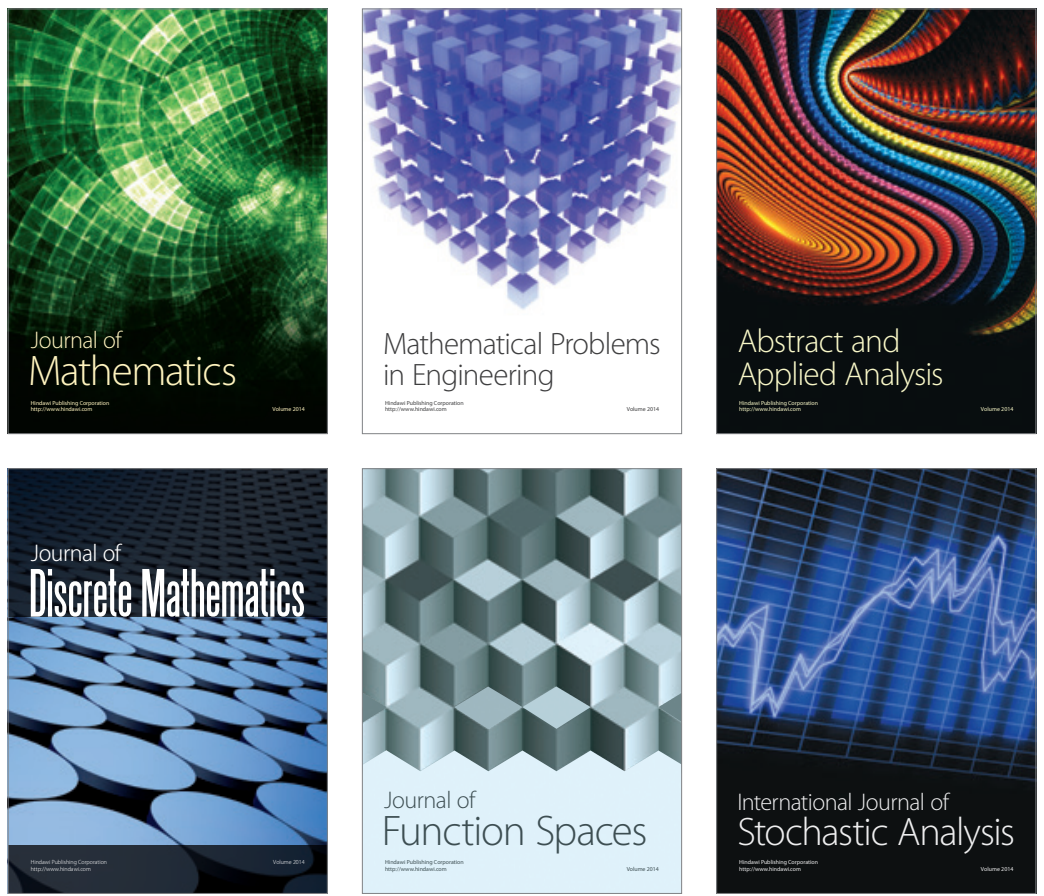

Journal of

Function Spaces

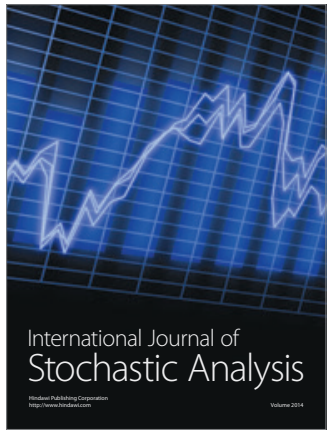

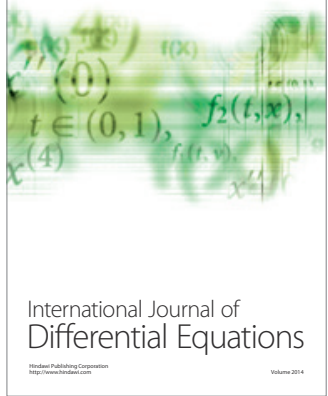
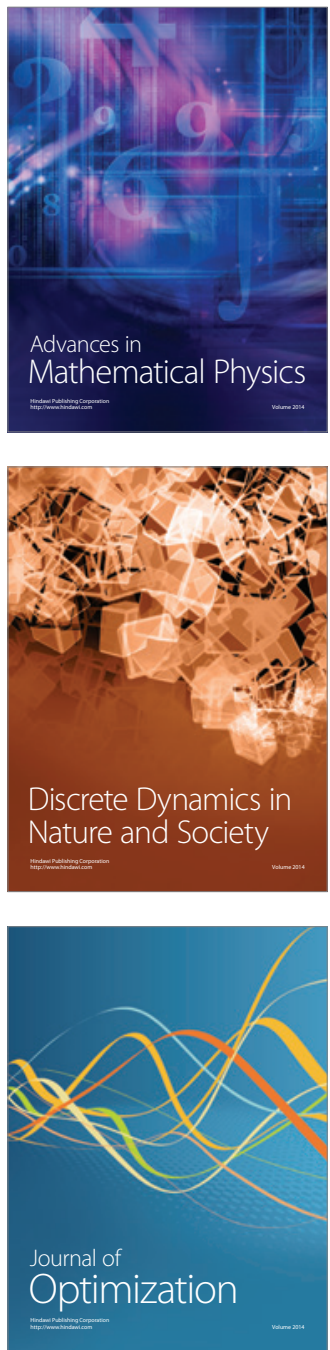